\title{
Damage Identification of Structures based on Wavelet Analysis and Artificial Fish-Swarm Algorithm
}

\author{
Deqing Guan ${ }^{1, a}$, Xiong Tang ${ }^{2, b}$,Jiawei Zhang ${ }^{3, c}$ \\ ${ }^{1,2,3}$ College of Civil Engineering, Changsha University of Science \& Technology, Changsha, China \\ a491596452@qq.com, b876812458@qq.com, c570716904@qq.com
}

\begin{abstract}
Keywords: wavelet analysis; artificial fish-swarm algorithm; damage identification; damage location; damage degree

Abstract. Wavelet analysis and artificial fish swarm algorithm are combined to study structural damage identification problem. Firstly, establishing the finite element model of structure and calculating the strain mode, then the wavelet maxima modulus of the wavelet transform are used to identify the damage location. On the basis of this, ,the objective function is constructed by the parameter of mode and frequency before and after the damage, and then by using the theory of artificial fish swarm algorithm to identify the damage degree of structures, establishing a structural damage identification method based on wavelet analysis and artificial fish swarm algorithm. It shows that the method is accurate and effective through the numerical simulation analysis of damage identification of a plane frame.This study has value for structural damage location and degree identification in engineering applications.
\end{abstract}

\section{Introduction}

In the construction period of the engineering structure, the damage of the structure can be caused by load and various unfavorable factors, which may cause structural damage in extreme cases. Therefore, the research of structural damage identification has important theoretical significance and engineering application value.

Wavelet transform is a time-frequency adaptive analysis method, it has the local analysis function, the signal can be found in other analysis methods can not be aware of the discontinuity and breakpoint, so people use wavelet transform to identify the damage of structure ${ }^{[1-2]}$. Zhang ${ }^{[3]}$ use wavelet transform to analyze the response signals of beams under moving loads, and achieve better recognition results. Guan ${ }^{[4]}$ proposed the wavelet transform method of corner mode to identify the damage of Timoshenko beam and elastic foundation beam. However, the accuracy of structural damage identification using wavelet analysis is not satisfactory. In recent years, with the development of related technologies, a group of intelligence algorithms have been put forward. The intelligent optimization algorithm is applied to the field of structural damage identification, which can better quantify the damage degree of structures. Moha ${ }^{[5]}$ applied genetic algorithm and particle swarm optimization to crack identification of structures. Xiaolei $\mathrm{Li}^{[6]}$ applied the fish-swarm algorithm to the optimization of complex power systems. $\mathrm{Li}^{[7]}$ applies artificial fish swarm algorithm to structural damage identification, proved that the artificial fish swarm algorithm is applied to the structural damage detection is feasible, But when the actual structure is complex, the computational efficiency is low. In this paper, the authors put forward the idea of combining wavelet analysis and intelligent algorithm to identify structural damage. By combining wavelet analysis and genetic algorithm, the damage of frame structure is successfully recognized.

Artificial fish swarm algorithm is a swarm intelligence algorithm that simulates the characteristics of fish stocks. The algorithm is easy to set up and easy to implement, and the convergence of the algorithm is not sensitive to the initial values of parameters. In this paper, the damage location of the structure is obtained by wavelet transform, and then the artificial fish swarm algorithm is used to determine the damage degree of the structure damage unit. A structural damage identification method is developed to identify the damage location and determine the damage degree. 


\section{Identification of Structural Damage Position Based on Wavelet Singularity Theory}

Wavelet Transform. For any function $x(t) \in L^{2}(R)$, the continuous wavelet transform is defined as:

$$
W_{x}(a, b)=\left(x, \Psi_{a, b}\right)=\int_{-\infty}^{+\infty} x(t) \psi_{a, b}^{*}(t) d t=\int_{-\infty}^{+\infty} x(t) \frac{1}{\sqrt{a}} \psi^{*}\left(\frac{t-b}{a}\right) d t .
$$

Where $a \in \mathrm{R}$ and $b \in \mathrm{R}$ are respectively scaling and translation parameters. For any function $x(t) \in L^{2}(R)$, the continuous wavelet transform is defined as:

$$
W_{x}(a, b)=\int_{-\infty}^{\infty} x(t) \psi_{a, b}^{*}(t) d t=\int_{-\infty}^{\infty} x(t) \frac{1}{\sqrt{a}} \psi^{*}\left(\frac{t-b}{a}\right) d t
$$

Principle of Wavelet Recognition for Damage Location. The singularity of the signal often occurs in the position where the structure is damaged. The damage of the structure can be attributed to the decrease of the structural stiffness at the cross section, Both sides of the injured areas should meet the following conditions:

Vertical displacement:

$$
\omega\left(v^{+}\right)=\omega\left(v^{-}\right) \text {. }
$$

Rotation:

$$
\frac{d \omega\left(v^{+}\right)}{d x}=\frac{d \omega\left(v^{-}\right)}{d x}
$$

Bending moment:

$$
E I\left(v^{+}\right) \frac{d^{2} \omega\left(v^{+}\right)}{d x^{2}}=E I\left(v^{-}\right) \frac{d^{2} \omega\left(v^{-}\right)}{d x^{2}} .
$$

Shearing force :

$$
E I\left(v^{+}\right) \frac{d^{3} \omega\left(v^{+}\right)}{d x^{3}}=E I\left(v^{-}\right) \frac{d^{3} \omega\left(v^{-}\right)}{d x^{3}} .
$$

The relationship between strain and rotation is:

$$
\varepsilon=K \frac{\partial \theta}{\partial x} \text {. }
$$

Where $w\left(v^{+}\right)$is the right vertical displacement, $w\left(v^{-}\right)$is the left vertical displacement, $\mathrm{x}$ is the coordinate of the length direction, $\theta$ is the rotation; $K$ is a constant.

The structure still satisfy both the compatibility conditions and internal force equilibrium conditions although that $E I\left(v^{+}\right)$and $E I\left(v^{-}\right)$at each side of damage cross section $\mathrm{V}$ are unequal. Because of the inequality of $E I\left(v^{+}\right)$and $E I\left(v^{-}\right)$, this result shows that the second derivative of the base frequency of simply-supported beam's damage cross section is discontinuous.Discontinuity of strain modal curve of damaged structure,therefore, the damage location of the structure can be identified by strain mode.

\section{Quantification of Structural Damage}

Artificial Fish-Swarm Algorithm. Artificial fish swarm algorithm is a random search optimization algorithm for simulating the behavior of fish stocks. Artificial fish swarm algorithm simulating natural fish behavior structure of artificial fish, by feeding, cluster and following behavior change their position, after a period of time the movement of artificial fish in the optimal value of aggregation, simple behavior through the artificial fish to find the global optimal value of the objective.

Artificial-Swarm Algorithm to determine the degree of damage. The intelligent algorithm is more relaxed for the objective function, such as differentiability of the objective function is not required, and has superior capability of global search. Artificial fish swarm algorithm as an emerging intelligent algorithm encompasses all of these advantages. In this paper, the natural vibration mode and natural frequency of the structure are taken as the parameters, and the objective 
function is established with the weighting and the minimum of the modal difference and the frequency difference before and after the damage:

$$
F=C_{\omega}\left(\sum_{i=1}^{m}\left(\frac{f_{i}^{\text {test }}-f_{i}^{c a l}}{f_{i}^{\text {test }}}\right)^{2}\right)^{\frac{1}{2}}+C_{\phi} \sum_{i=1}^{n}\left(\sum_{j=1}^{k}\left(\phi_{i j}^{\text {test }}-\phi_{i j}^{\text {cal }}\right)^{2}\right)^{\frac{1}{2}} .
$$

Where $C_{\omega}$ and $C_{\phi}$ are weighting factors, $\phi_{i j}^{\text {test }}$ is the displacement of the $\mathrm{j}$-th node of the $\mathrm{i}$-th order natural mode with the damage structure of the finite element simulation, $\phi_{i j}^{c a l}$ is the displacement of the $\mathrm{j}$-th node in the $\mathrm{i}$-th order natural mode of the corresponding damage state in the search process of the fish group algorithm, $f_{i}^{\text {test }}$ is the $\mathrm{i}$-th order natural frequency of the damaged structure with finite element simulation, $m$ is the natural frequency order of extraction, $n$ is the natural mode order of extraction, $\mathrm{k}$ is the number of nodes of structural finite element model.

Considering that the natural mode is more sensitive to the error than the natural frequency, $C_{\omega}=0.4, C_{\phi}=0.6$ in this paper. The unit damage size $\alpha_{i}$ (Damage size of unit $\mathrm{i}$ and $0 \leq \alpha_{i}<1$ ) in the finite element model is used as the search space for the fish algorithm. Each of the damage conditions $\alpha$ can obtain the corresponding natural frequency $f_{i}^{c a l}$ and the vibration mode $\phi_{i j}^{c a l}$. The data of the natural frequency n-order mode of the vibration m-order mode is substituted into the objective function F. Its value is the fitness value of the corresponding damage condition. When the fitness value reaches the preset accuracy or the number of iterations exceeds the preset maximum number of iterations, the degree of damage of the corresponding unit that makes the fitness value reach the minimum value is the degree of damage of each damage unit.

\section{Numerical Simulation Analysis}

Structural Damage Conditions. This frame structure uses $Q 235$ steel, the length of the bea $\mathrm{m}$ and column is $3000 \mathrm{~mm}$, cross section size: $300 \times 500 \mathrm{~mm}$, elastic modulus: $2.1 \times 10^{11} \mathrm{~N} / \mathrm{m}^{2}$, densi ty: $\rho=7800 \mathrm{~kg} / \mathrm{m}^{3}$,Poisson ratio: $\mu=0.3$. and assuming that this frame structure has a $5 \%, 7 \%$, $3 \%$ damage at units $15,35,50$, respectively. the model of the frame structure is shown in $\mathrm{Fi}$ g.1.

Identify the Damage Location of the Frame Structure. First of all, the finite element model of frame structure is established.The damage of the structure is simulated by the method of reducing the stiffness of the element. Then the strain modal is obtained by dynamic analysis of the structure. The wavelet coefficients are obtained by db1 wavelet transform, as shown in figure 2 . As you can see from the graph, the wavelet coefficients are mutated at units 15 th, 35th, and 50th, indicating that the structure is damaged in these 3 places.

Quantify the Damage to the Frame Structure. As we can see above, the structures are damaged only at units 15,35 and 50, On this basis, the objective function is constructed by using the first 3 natural frequencies and vibration modes before and after structural damage, and then the target function of fish swarm algorithm is used to search the damage degree of the damage. The recognition result is shown in Figure 3, and the optimization curve is shown in figure 4. The relative error of the damage degree of the three damage units is less than $3 \%$, which shows that fish swarm algorithm can effectively quantify the degree of structural damage.

\section{Conclusions}

1. Based on wavelet analysis theory and artificial fish swarm algorithm, a wavelet fish swarm algorithm which can identify the damage location and identify the damage degree is established.

2. Firstly, the damage location is identified by wavelet analysis method, and then the artificial fish swarm algorithm is used to identify the damage degree at the injured position. The damage position 


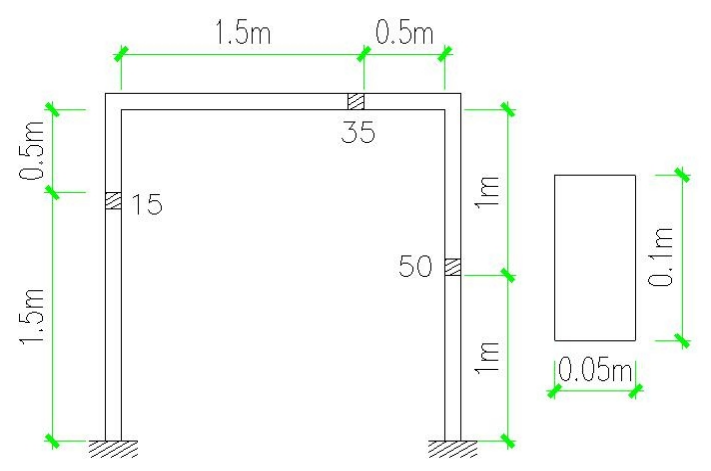

Fig.1. Model of frame structure

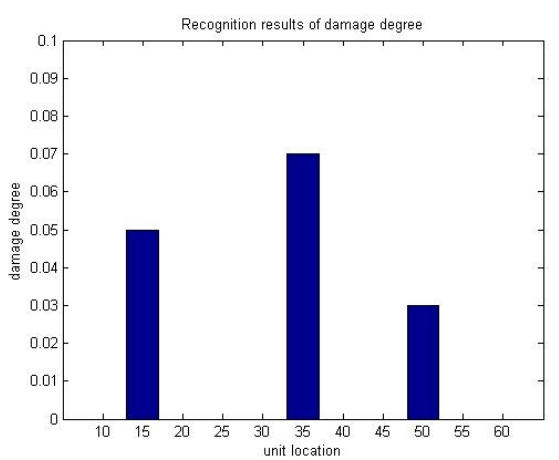

Fig.3. Quantification of damage degree

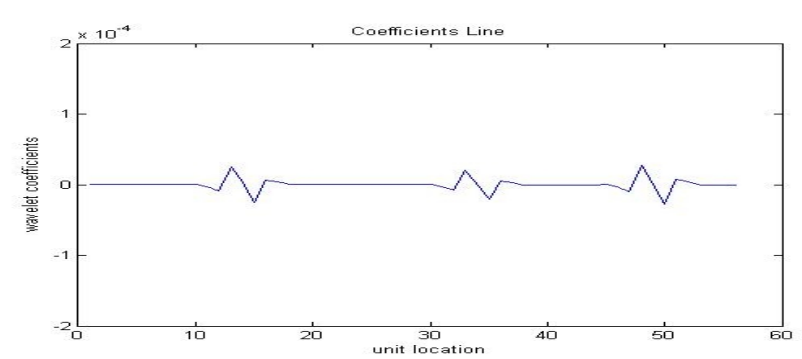

Fig.2. Wavelet coefficient

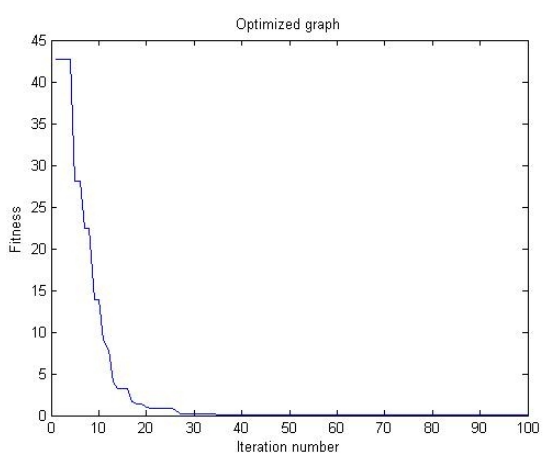

Fig.4. Optimization of Fish-swarm algorithm

and the degree of damage can be identified by a small amount of calculation.

3. The method of damage identification using intelligent algorithm is large in amount of calculation and low in recognition of small damage, The method of wavelet analysis and fish swarm algorithm combined with less than 5\% small damage identification accuracy is also higher.

4. In this paper, the identification of damage location and damage degree of planar frames is taken as an example to verify the effectiveness of the proposed method. Its principle can be widely applied to the damage identification of other complex bending structures, so it can be used as a reference for engineering applications.

\section{Acknowledgements}

This work was financially supported by the National Natural Science Foundation of China (51378079).

\section{References}

[1] Nematollahi M A,Farid M,Henatiyan M R,et al:.Journal of Aerospace Engineering(Procee -dings of the Institution of Mechanical Engineers,Part G) Vol.226(2012), p.1243

[2] Xijun Liu,Linjie Xiang,Suxia Zhang:Journal of vibration measurement and diagnosis Vol. 35(2015), p.866

[3] Zhang W,Wang Z,Ma H: Key Engineering Materials Vol.413(2009), p.285

[4] Deqing Guan,Xin Jiang: Journal of vibration and shock Vol.25(2007), p.67

[5] Moha S C.Yadav amit and maiti dipak kumar:Engineering Computations Vol.31(2014), p. 1514

[6] Xiaolei Li, Jixin Qian: Journal of circuits and systems Vol.3(2003), p.1

[7] Cheng Li,Ling Yu:Journal of vibration and shock Vol.38(2014), p.113 